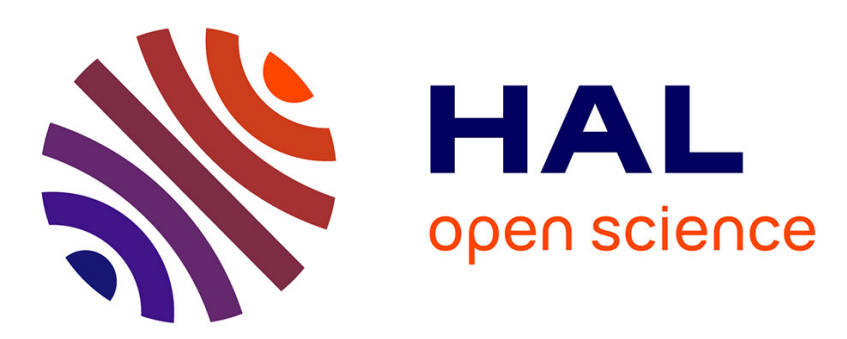

\title{
AIRCRAFT PARAMETER AND DELAY IDENTIFIABILITY
}

Carine Jauberthie, Lotfi Belkoura, Lilianne Denis-Vidal

\section{To cite this version:}

Carine Jauberthie, Lotfi Belkoura, Lilianne Denis-Vidal. AIRCRAFT PARAMETER AND DELAY IDENTIFIABILITY. European Control Conference, Sep 2003, Cambridge, United Kingdom. hal01966357

\section{HAL Id: hal-01966357 https://hal.laas.fr/hal-01966357}

Submitted on 28 Dec 2018

HAL is a multi-disciplinary open access archive for the deposit and dissemination of scientific research documents, whether they are published or not. The documents may come from teaching and research institutions in France or abroad, or from public or private research centers.
L'archive ouverte pluridisciplinaire $\mathbf{H A L}$, est destinée au dépôt et à la diffusion de documents scientifiques de niveau recherche, publiés ou non, émanant des établissements d'enseignement et de recherche français ou étrangers, des laboratoires publics ou privés. 


\author{
C. Jauberthie* ${ }^{*}$ L. Belkoura ${ }^{\dagger}$, L. Denis-Vidal ${ }^{\dagger}$ \\ * University of Technology Compiègne, BP 20 529, 60205 Compiègne, France, \\ e-mail: carine.jauberthie@utc.fr \\ fax: +33 344234477 \\ ${ }^{\dagger}$ University of Sciences and Tech. Lille, 59655 Villeneuve d'Ascq, France, \\ e-mail: lotfi.belkoura@univ-lillel.fr, denvideattglobal.net
}

Keywords: Nonlinear systems, Time-delay systems, Identifiability, Linearization, Differential algebra.

\begin{abstract}
This paper considers the identifiability problem for a model given by aerospace domain describing aircraft dynamics with time delays. Analytic and algebraic approaches are used to show how different approximations derived from the original non linear and retarded system may result in different identifiability conclusions.
\end{abstract}

\section{Introduction}

System identification based on physical laws often involves parameter estimation. Before performing estimation problem, it is necessary to investigate its identifiability. It is a mathematical and a priori problem. This paper is concerned with the identifiability of a model given by aerospace domain describing aircraft dynamics. This model is derived from the general equations of motion:

$$
\left\{\begin{array}{rl}
m \frac{d V}{d t} & =\sum F_{e} \\
\frac{d \mathcal{C}}{d t} & =\sum M_{e}
\end{array},\right.
$$

where $\frac{d V}{d t}$ denotes the acceleration of the gravity center, $\mathcal{C}$ the kinetic moment, $\sum F_{e}$ the sum of the forces acting on the aircraft and $\sum M_{e}$ the moment of these forces. In order to improve the model accuracy, the input which consists here in the turbulence, is supposed to act at three different points of the fuselage. Let $\sigma_{i}$ denote the shift operator associated to $\tau_{i}>0$, and defined for some function $w(t)$ by:

$$
\left(\sigma_{i} w\right)(t):=w\left(t-\tau_{i}\right), \quad i=1, . ., 3,
$$

and let also $\tau_{3}=\tau_{2}-\tau_{1}$. The projection of equations (1) on the aerodynamic reference frame of the aircraft yields the following non linear and retarded equations [4]:

$$
\sum^{p}\left\{\begin{aligned}
\dot{V}= & k_{0} \sin (\theta-\alpha)+V^{2}\left(k_{1}+k_{2} \alpha\right) \\
V(\dot{\theta}-\dot{\alpha})= & k_{0} \cos (\theta-\alpha)+k_{3} q V+V^{2}\left[k_{4}+\right. \\
& p_{1}(\alpha+u)+p_{2}\left(\alpha+\sigma_{1} u\right)+ \\
& \left.p_{3}\left(\alpha+\sigma_{2} u\right)+p_{3} p_{4}\left(\sigma_{3} \alpha+\sigma_{2} u\right)\right] \\
\dot{q}= & k_{5} q V+V^{2}\left[k_{6}+p_{5}(\alpha+u)+\right. \\
& p_{6}\left(\alpha+\sigma_{1} u\right)+p_{7}\left(\alpha+\sigma_{2} u\right)+ \\
& \left.p_{4} p_{7}\left(\sigma_{3} \alpha+\sigma_{2} u\right)\right] \\
\dot{\theta}= & q,
\end{aligned}\right.
$$

where $V$ denotes the speed of the aircraft, $\alpha$ the angle of attack, $\theta$ the pitch angle, $q$ the pitch rate, and $u$ the input. The set of parameters $k_{i}, i=0, \ldots, 6$ is assumed a priori known (here for instance $k_{0}=g$, the gravity coefficient) and doesn't need to be estimated. The state vector is defined by:

$$
x:=(V, \alpha, q, \theta)^{T}
$$

and is also assumed available from measurements. In this paper we are interested in the identifiability of the set:

$$
p=\left(\underline{p}, \tau_{1}, \tau_{2}\right) \quad \text { with } \quad \underline{p}=\left(p_{1}, \ldots, p_{7}\right) .
$$

The vector $p$ is assumed in some subset $\Omega$ of $\mathbb{R}^{9}$, and the main problem in our case comes from the introduction of the delays in the set of unknown parameters. To our knowledge, there is no existing method for solving the identifiability problem for general non linear and retarded systems with a priori unknown delays.

The aim of this paper is to show how different approximations derived from the original non linear and retarded system may result in different identifiability conclusions. The presentation is organized as follows: Section 2 presents an analytic approach based on the linearization of the model in the vicinity of a nominal trajectory. An algebraic approach is developed in Section 3, where the nonlinearities are maintained while the retarded terms are assumed known or approximated using Taylor expansion or Padé approximation. Section 4 presents some concluding remarks. Most of the computations related to the aircraft models are implemented in Maple, a symbolic computation language.

\section{Analytic approach}

\subsection{Identifiability of time-delay systems}

The result presented in this section is mainly derived from the (non) regularity of the impulse response [1]. Consider the following linear time delay system:

$$
T^{Q}: \dot{x}(t)=\sum_{i=0}^{r} A_{i} x\left(t-\tau_{i}\right)+B_{i} u\left(t-\tau_{i}\right),
$$

where $0 \leq \tau_{1}<\ldots<\tau_{r}, x(t) \in \mathbb{R}^{n}$ and $u(t) \in \mathbb{R}^{m}$. The above equation is subject to an initial condition $x_{0}(\theta)=\phi(\theta)$, $-\tau_{r} \leq \theta \leq 0$, which is assumed sufficiently smooth. The set of 
unknown parameters is given here by the coefficient matrices and time delays:

$$
Q=\left\{A_{1}, . . A_{r}, B_{1}, . . B_{r}, \tau_{1}, . . \tau_{r}\right\}
$$

Equation (6) is known to have a unique solution given any locally integrable $u($.$) [2]. Denoting x(Q, u)$ a solution of (6) for a given $Q$ in some set $\Omega$ and any sufficiently smooth initial condition $x_{0}$, the problem of identifiability is stated as follows:

Definition 1 The system $T^{Q}$ is said to be (globally) identifiable at $Q$ with respect to $\Omega$ if there exists an input $u$ such that, for any $Q^{*} \in \Omega$, the equality $Q=Q^{*}$ results from $x(Q, u)=x\left(Q^{*}, u\right)$.

Similarily, local identifiability can be defined so that $T^{Q}$ is said to be locally identifiable at $Q$ if there exists a neighborhood $W$ in $\Omega$ such that the system is globally identifiable with respect to $W$. Furthermore, in most models there exist atypical points in $\Omega$ where the model is unidentifiable. Therefore, the previous definition can also be generically extended so that $T^{Q}$ is said to be (globally) structurally identifiable if it is (globally) identifiable at all $Q \in \Omega$ except at the points of a subset of zero measure in $\Omega$. Now for $s \in \mathbb{C}$, let us define the entire (matrix-valued) functions:

$$
\begin{gathered}
A(s)=\sum_{i=0}^{r} A_{i} e^{-\tau_{i} s}, \quad B(s)=\sum_{i=0}^{r} B_{i} e^{-\tau_{i} s} \\
\langle A(s), B(s)\rangle=\left[B(s), A(s) B(s), \ldots, A^{n-1}(s) B(s)\right] .
\end{gathered}
$$

Theorem 2 [1] Assume that, for some $s \in \mathbb{C}$,

$$
\operatorname{rank}\langle A(s), B(s)\rangle=n \text {. }
$$

Then the time delay system $T^{Q}$ is (structurally globally) identifiable.

\subsection{Application to the linearized aircraft dynamic}

The considered airplane dynamics (3) can be written in the equivalent from:

$$
\Sigma^{p}: \dot{x}=f\left(x, \sigma_{3} x, u, \sigma_{1} u, \sigma_{2} u, p\right)
$$

for some appropriate non linear function $f$. From (3), one can easily check that the obtained function $f$ is continuously differentiable w.r.t. all its arguments. The time invariant linearized model is obtained assuming the knowledge of a nominal and constant trajectory (i.e. $x(t)=x_{e}, u(t)=u_{e}$, available for instance from the system's history before the turbulent atmosphere). If $y(t)$ and $w(t)$ denote the state and control perturbations, linearization of (11) results in the time delay system:

$$
\Upsilon^{p}: \dot{y}(t)=\sum_{i=0}^{3} A_{i}(\underline{p}) y\left(t-\tau_{i}\right)+B_{i}(\underline{p}) w\left(t-\tau_{i}\right)
$$

where the matrices of constant coefficients are given by [9]:

$$
\begin{aligned}
& A_{i}(\underline{p})=\left.\nabla_{x\left(t-\tau_{i}\right)} f(., p)\right|_{e}, \\
& B_{i}(\underline{p})=\left.\nabla_{u\left(t-\tau_{i}\right)} f(., p)\right|_{e},
\end{aligned}
$$

and $\left.\nabla_{z\left(t-\tau_{i}\right)} f(., p)\right|_{e}$ is the Jacobian matrix of $f$ with respect to $z\left(t-\tau_{i}\right)$ (for ease of notations, we have let $\tau_{0}:=0$ ). The identifiability of the linearized system $\Upsilon^{p}$ can be formulated in the same terms as Definition 1, except that we are concerned here with the set $p=\left(p, \tau_{1}, \tau_{2}\right)$ rather than $Q$ in (7). However, it is clear that identifiability of the system $T^{Q}$ at $Q$ implies that of $\Upsilon^{p}$ at $p$ if one shows that the map:

$$
p=\left(\underline{p}, \tau_{1}, \tau_{2}\right) \rightarrow Q(p)
$$

where

$$
Q(p)=\left[A_{0}(\underline{p}), \ldots, A_{3}(\underline{p}), B_{0}(\underline{p}), \ldots, B_{3}(\underline{p}), \tau_{1}, . ., \tau_{3}\right]
$$

is injective. Since $\tau_{3}=\tau_{2}-\tau_{1}$, the delay case is obvious and it remains to show that the knowledge of the coefficient matrices $A_{i}(\underline{p})$ and $B_{i}(\underline{p})$ uniquely determines the set $\underline{p}=\left(p_{1}, \ldots, p_{7}\right)$. Using the equilibrium point equations, a straightforward computation of the nonzero entries of $A_{1}, B_{0}, B_{1}$ and $B_{2}$, yields the reduced matrix:

$$
Q_{r}(\underline{p})=\left(\begin{array}{cccc}
-V_{e} p_{3} p_{4} & -V_{e} p_{1} & -V_{e} p_{2} & -V_{e} p_{3}\left(1+p_{4}\right) \\
V_{e}^{2} p_{7} p_{4} & V_{e}^{2} p_{5} & V_{e}^{2} p_{6} & V_{e}^{2} p_{7}\left(1+p_{4}\right)
\end{array}\right)
$$

where $V_{e}$ is the nominal speed of the aircraft. It is easily seen from (17) that

$$
Q_{r}(\underline{p})=Q_{r}\left(\underline{p}^{*}\right) \Rightarrow \underline{p}=\underline{p}^{*}
$$

and hence the map $Q$ in (16) is injective. Therefore, and by virtue of Theorem 2, identifiability of $\Upsilon^{p}$ is ensured at $p$ if, for some $s \in \mathbb{C}$,

$$
\operatorname{rank}\langle A(p, s), B(p, s)\rangle=n,
$$

where

$$
A(p, s)=\sum_{i=0}^{3} A_{i}(\underline{p}) e^{-\tau_{i} s}, B(p, s)=\sum_{i=0}^{3} B_{i}(\underline{p}) e^{-\tau_{i} s} .
$$

The use of the symbolic computation language Maple gives $\operatorname{rank}\langle A(p, 0), B(p, 0)\rangle=4$ which shows the structural global identifiability of the linearized system.

Unlike the free delay case where the properties one can obtain from the linearized system still hold for the original plant in the vicinity of the linearization point, the extension of such result to the retarded case is still an open problem. The linearized aircraft model is identifiable but it is not known whether or not the identifiability of the linearization implies that of the nonlinear model with delay. 


\section{Algebraic approach}

\subsection{Input-output approach for testing Identifiability}

The input-output approach can be used to test identifiability of some non linear systems. This method is based on differential algebra [7] and consists in rewritting, when it is possible, the non linear system as a differential polynomial system that will be completed with $\dot{p}=0$ where $p$ is the vector of parameters of the system. The resulting system $\Gamma^{p}$ can be described by the following polynomial system:

$$
\Gamma^{p}\left\{\begin{array}{l}
R(\dot{x}, x, u, \dot{u}, p)=0, \\
S(x, y, p)=0, \\
\dot{p}=0 .
\end{array}\right.
$$

The notion of identifiability is strongly connected to observability. In the 90s Fliess and Diop propose a new approach of nonlinear observability and identifiability, based on differential algebra [6]. The initial conditions are ignored as they are in the model $\Gamma^{p}$. A solution of $\Gamma^{p}$ is a triplets of functions $(x, y, u)$ which satisfies all the equations of the model. Thus, the solution of these equations may not be unique and some solutions may be degenerate. Therefore the set of non-degenerate solutions $\bar{x}(p, u), \bar{y}(p, u)$, corresponding to every possible initial condition, have to be involved in the identifiability definition. Here we adopt the definition introduced in [8].

Definition 3 The model $\Gamma^{p}$ is globally identifiable with respect to $\Omega$ at $p$ iffor any $p^{*} \in \Omega, p^{*} \neq p$ there exists a control $u$, such that $\bar{y}(p, u) \neq \emptyset$ and $\bar{y}(p, u) \cap \bar{y}(\tilde{p}, u)=\emptyset$.

The previous definition is well in line with the usual formulation [10] which consider initial conditions: If $\bar{y}(p, u)=$ $\bar{y}\left(p^{*}, u\right)$ then $p=p^{*}$. As in the previous section, local and structural identifiability can also be defined in the same manner. In this approach $I$ is the radical of the differential ideal generated by the equations of $\Gamma^{p}$ and the ranking:

$$
[p] \prec[y, u, \dot{u}] \prec[x]
$$

is chosen in order to eliminate the state variables. In general, $I$ should be written as the intersection of regular differential ideals which admit a characteristic presentation. A characteristic presentation [3] is a set of polynomials which is a canonical representant of the ideal. Generally several characteristic presentations are obtained but only one gives general input-output polynomials which allow us to analyze identifiability of parameters. The others correspond to particular cases of value of parameters or degenerate solutions. The method is validate by checking the independence of some monomials in $y, u$ and their derivatives occuring in the input-output polynomials.

The computation are achieved with an algorithm implemented in Maple [5]. It is based on the Rosenfeld-Groebner algorithm which has been realized and implemented by F. Boulier in the package Diffalg [3].

\subsection{The case of known delays}

In case of a priori known delays $\tau_{1}$ and $\tau_{2}$, the data $x\left(t-\tau_{i}\right)$ and $u\left(t-\tau_{i}\right)$ become available and it is easy to show that $\Sigma \underline{\underline{p}}$ is globally identifiable at $p$. First, it is clear from (3) that the identifiablity of $\underline{p}$ (as well as that of $p$ ) only depends on the second and third equations. Let us define $F_{1}(x, \dot{x})$ and $F_{2}(x, \dot{x})$ such that:

$$
\begin{aligned}
V^{2} F_{1}(x, \dot{x})= & V(\dot{\theta}-\dot{\alpha})-k_{0} \cos (\theta-\alpha) \\
& -k_{3} q V-V^{2} k_{4}, \\
V^{2} F_{2}(x, \dot{x})= & \dot{q}-k_{5} q V-V^{2} k_{6}
\end{aligned}
$$

This allows us to rewrite the original problem in the polynomial form (21) as:

$$
\Sigma^{\underline{p}}\left\{\begin{aligned}
w_{1}= & p_{1}(\alpha+u)+p_{2}\left(\alpha+\sigma_{1} u\right) \\
& +p_{3}\left(\alpha+\sigma_{2} u\right)+p_{3} p_{4}\left(\sigma_{3} \alpha+\sigma_{2} u\right) \\
w_{2}= & p_{5}(\alpha+u)+p_{6}\left(\alpha+\sigma_{1} u\right) \\
& +p_{7}\left(\alpha+\sigma_{2} u\right)+p_{4} p_{7}\left(\sigma_{3} \alpha+\sigma_{2} u\right) \\
y_{1}= & w_{1}, y_{2}=w_{2}, y_{3}=\alpha, y_{4}=\dot{\alpha}, \dot{p}=0
\end{aligned}\right.
$$

where $w_{1}=F_{1}(x, \dot{x}), w_{2}=F_{2}(x, \dot{x}), \alpha$ and $\dot{\alpha}$ are measured outputs which are equal respectively to $y_{1}, y_{2}, y_{3}$ and $y_{4}$. When $\tau_{1} \neq \tau_{2}$, the algorithm described in section 3.1 returns the following exhaustive summary from which the global identifiability of $p$ is clear:

$$
\left\{p_{1}, p_{2}, p_{3}, p_{3} p_{4}, p_{5}, p_{6}, p_{7}, p_{4} p_{7}\right\} .
$$

Now, when the delays are unknown, the presented approach is unusable unless one approximates the retarded terms. Among all possible approximations, expansion in Taylor series and Padé approximants are selected.

\subsection{Taylor expansion}

Approximation of the shift operator $\sigma_{i}$ in (2) by a expansion in Taylor serie at first order has the form:

$$
\left(\sigma_{i} w\right)(t) \approx w(t)-\tau_{i} \dot{w}(t), i=1,2,3 .
$$

Using the previous considerations, the original problem is transformed into the the polynomial form:

$$
T^{p}\left\{\begin{array}{c}
w_{1}=p_{1}(\alpha+u)+p_{2}\left(\alpha+u-\tau_{1} \dot{u}\right) \\
\quad+p_{3}\left(\alpha+u-\tau_{2} \dot{u}\right)+p_{3} p_{4}\left(\alpha+u-\tau_{3} \dot{\alpha}-\tau_{2} \dot{u}\right), \\
w_{2}=p_{5}(\alpha+u)+p_{6}\left(\alpha+u-\tau_{1} \dot{u}\right) \\
\quad+p_{7}\left(\alpha+u-\tau_{2} \dot{u}\right)+p_{4} p_{7}\left(\alpha+u-\tau_{3} \dot{\alpha}-\tau_{2} \dot{u}\right), \\
y_{1}=w_{1}, y_{2}=w_{2}, y_{3}=\alpha, y_{4}=\dot{\alpha}, \dot{p}=0
\end{array}\right.
$$

where $w_{1}=F_{1}(x, \dot{x}), w_{2}=F_{2}(x, \dot{x}), \alpha$ and $\dot{\alpha}$ are measured outputs. The previous algorithm yields one characteristic presentation which contains two input-output polynomials in $y_{1}$, $y_{2},\left(y_{3}+u\right), \dot{u}$ and $y_{4}$, and the following exhaustive summary:

$$
\begin{aligned}
& \left(p_{1}+p_{2}+p_{3}+p_{3} p_{4}\right),\left(p_{2} \tau_{1}+p_{3} \tau_{2}+p_{3} p_{4} \tau_{2}\right), p_{3} p_{4} \tau_{3}, \\
& \left(p_{5}+p_{6}+p_{7}+p_{7} p_{4}\right),\left(p_{6} \tau_{1}+p_{7} \tau_{2}+p_{7} p_{4} \tau_{2}\right), p_{7} p_{4} \tau_{3} .
\end{aligned}
$$


We can just notice here that 6 terms are given for the identifiability of 9 parameters. Therefore, the approximated system $T^{p}$ is neither globally nor locally identifiable. Moreover, even in case of a priori known delays, the linearization (27) resulted in a loss of identifiability of the original plant $\Sigma \underline{\underline{p}}$.

\subsection{Padé approximants}

The delay expression in frequency domain is given by an exponential function which is approached with Padé approximants at first order by $e^{-s \tau_{i}} \approx\left(1-\frac{s \tau_{i}}{2}\right) /\left(1+\frac{s \tau_{i}}{2}\right)$. In the time domain, this yields the differential equations:

$$
\left(\sigma_{i} w\right)(t)+\frac{\tau_{i}}{2}\left(\dot{\sigma_{i}} w\right)(t)=w(t)-\frac{\tau_{i}}{2} \dot{w}(t), i=1,2,3
$$

With this approximation, one has to define new state variables $z_{i}(t), i=1,2,3$ which consists in the unknown terms $\sigma_{1} u, \sigma_{2} u$, and $\sigma_{3} \alpha$. The set of polynomial is now given by:

$$
D^{p}\left\{\begin{aligned}
w_{1}= & p_{1}(\alpha+u)+p_{2}\left(\alpha+z_{1}\right)+p_{3}\left(\alpha+z_{2}\right)+ \\
& p_{3} p_{4}\left(z_{2}+z_{3}\right) \\
w_{2}= & p_{5}(\alpha+u)+p_{6}\left(\alpha+z_{1}\right)+p_{7}\left(\alpha+z_{2}\right)+ \\
& p_{4} p_{7}\left(z_{2}+z_{3}\right) \\
\tau_{1} \dot{z_{1}}= & 2\left(-z_{1}+u\right)-\tau_{1} \dot{u} \\
\tau_{2} \dot{z_{2}}= & 2\left(-z_{2}+u\right)-\tau_{2} \dot{u} \\
\tau_{3} \dot{z_{3}}= & 2\left(-z_{3}+\alpha\right)-\tau_{3} \dot{\alpha}, \\
y_{1}= & w_{1}, y_{2}=w_{2}, y_{3}=\alpha, y_{4}=\dot{\alpha}, \dot{p}=0 .
\end{aligned}\right.
$$

The previous algorithm gives the general characteristic presentation which contains two input-output polynomials which are too large to be written here. The exhaustive summary of the parameters in the set $p$ is analyzed with the same algorithm which conclude to the structural global identifiability of $D^{p}$.

\section{Conclusion}

This paper presents some approaches for the parameters and delays identifiability of aircraft dynamics which consist in a non linear and retarded system. We have shown that if the delays are known, the nonlinear model is identifiable. In case of unknown delays, the classical methods to test identifiability can't be applied. More importantly, it is shown how different approximated models can lead to opposite results. An approximation of the delays based on Taylor'expansion at first order yields a model which is neither globally nor locally identifiable, while the Pade approximants, as well as a linearization of the model in the vicinity of a nominal trajectory lead to a structurally identifiable system. New results showing to what extend the properties one can obtain from an approximated system still hold for the original nonlinear and retarded ar still expected. However, under some appropriate assumptions, the identifiable approximated models could be used to estimate the parameters and the delays. Another open problem concerns robustness since, throughout this paper we have assumed perfect data.

\section{References}

[1] L. Belkoura, Y Orlov. "Identifiability analysis of linear delay-differential systems", IMA Journal of Math. Control and Information, 19, 73-81, (2002).

[2] R. Bellman, K. E. Cooke. 'Differential-difference equations", Academic Press, New York (1963).

[3] F. Boulier, D. Lazard, F. Ollivier, M. Petitot. "Computing representations for the radicals of a finitely generated differential ideals", Technical report, LIFL, Université Lille I.

[4] P. Coton. "Validation de modèles de représentation du comportement des aérodynes en rafales verticales", Rapport technique IMFL 81/20 (1981).

[5] L. Denis-Vidal, G. Joly-Blanchard, C. Noiret, M. Petitot. "An algorithm to test identifiability of non-linear systems", Proc. 5th IFAC NOLCOS, St Petersburg, Russia pp 174-178 (2001).

[6] S, Diop, M. Fliess. "Nonlinear observability, identifiability, and persistent trajectories", Proc. 30th CDC, Brighton, pp 714-719 (1991).

[7] E.R. Kolchin. "Differential algebra and algebraic groups", Academic Press, New York.

[8] L. Ljung, T. Glad. "On global identifiability for arbitrary model parametrizations", Automatica 30-2, pp 265-276 (1994).

[9] M. Malek-Zavarei. "Time-delay systems, Analysis, Optimization and Applications", North-Holland Systems and Control Series, 9, 1987.

[10] E. Walter. "Identifiability of state space models", Lecture Notes Biomath, 46, (1982). 